NUCLEAR POWER

Doesn't have to cost the earth Energy Policy 91, 371-382 (2016)

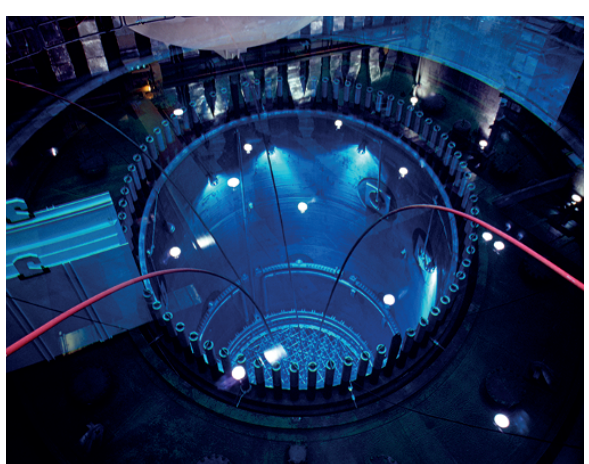

The high costs associated with the construction of nuclear power plants, among other factors, impede their large-scale deployment. This is exacerbated by analyses of historical cost data up to the 1980s for plants built by early-adopters - France and the US - that suggest an inevitable trend of escalating costs over time. Now, Jessica Lovering and colleagues at The Breakthrough Institute in California and Carnegie Mellon University perform a comprehensive analysis of construction cost data for seven countries, covering $58 \%$ of global reactors built between 1954 and 2015, and conclude that an escalating cost trend is not intrinsic to nuclear technology, but instead depends on country, institutional factors and technological maturity.

Overnight construction costs (OCCs) make up around 55\% of the total levelized cost of electricity generation for nuclear. They are reactor-specific and include engineering and construction costs, as well as land and site costs. The researchers analysed OCC data for 349 reactors in the US, France, Canada, West Germany, Japan, India and South Korea. They found that, while costs had sharply increased for reactors in the US, other countries (like France and Canada) experienced a milder cost escalation, and South Korea even saw a decrease in OCCs over time. Lower costs might have derived from design standardization and parallel construction of multiple reactors on the same site. The researchers suggest that projections of future costs that inform decision makers should take into account the observed variance in historical trends.

\section{ENERGY STORAGE}

\section{The best of both worlds}

Adv. Energy Mater. http://doi.org/§3kn3b (2016)

Lithium-ion batteries can store a large amount of energy but often don't deliver the energy quickly. Supercapacitors, on the other hand, have high power densities but suffer from limited energy densities. Lithium- or sodium-ion capacitors, which combine the advantages of the two devices, have emerged as a promising way to achieve both high energy and high power performance. Although sodium-ion capacitors are costeffective compared with their lithium counterparts, the slow ionic diffusion and reaction kinetics are the main challenges. Yun-Sung Lee and colleagues at Chonnam National University, University of Western Ontario and Seoul National University have now reported a sodium-ion capacitor that mitigates the problem of the sluggish kinetics

Community, co-operative, citizen and municipal energy projects mostly focus on renewables and make a small but increasing contribution to the energy mix. Such schemes could play an important role in the transition to a low-carbon energy system, but a greater understanding of the necessary financial and regulatory support is required to help expand their number. Towards that end, Stephen Hall at the University of Leeds and colleagues in the UK compared the role of financial institutions in Germany and the UK to see how their different frameworks affect civic energy ownership.

The researchers analysed policy and statistical publications, and interviewed 36 people from across the UK and German electricity sectors, including utility executives, finance providers, and project developers. They found that the UK's centralized, market-based financial system makes it difficult for financial institutions to capitalize small-scale renewable projects. As such, the electricity system only incorporates small amounts of civic ownership and future participation is likely to be limited. Meanwhile, Germany's decentralized system of local banking institutions provides greater support and favourable loans to small and medium renewables schemes. Common governing principles and values between financial institutions and civic energy schemes were also deemed important to the schemes' success. The findings demonstrate the importance of the economic context and path-dependence of national financial institutions in supporting the growth of the civic energy sector.

and exhibits an energy density of $118 \mathrm{~W} \mathrm{~h} \mathrm{~kg}^{-1}$ and a power density of $850 \mathrm{~W} \mathrm{~kg}^{-1}$ that even outperforms the lithium-ion capacitors.

The sodium-ion capacitor was built with a carbon-coated sodium conductor $\left(\mathrm{Na}_{3} \mathrm{~V}_{2}\left(\mathrm{PO}_{4}\right)_{3}\right)$ and a carbon material derived from cinnamon sticks as electrodes. The porous characters of $\mathrm{Na}_{3} \mathrm{~V}_{2}\left(\mathrm{PO}_{4}\right)_{3}$ and the bio-derived carbon are key in enhancing the sodium insertion/de-insertion in $\mathrm{Na}_{3} \mathrm{~V}_{2}\left(\mathrm{PO}_{4}\right)_{3}$ as well as the adsorption/desorption on the electrode surfaces, both of which contribute to the high energy and power densities. In addition, $\mathrm{Na}_{3} \mathrm{~V}_{2}\left(\mathrm{PO}_{4}\right)_{3}$ can retain its framework structure well during the electrochemical reactions, which is mainly responsible for the observed high stability in cycling experiments.

\section{FUEL CELLS}

\section{Illuminating deactivation}

\section{Angew. Chem. Int. Ed. 55, 3086-3090 (2016)}

Fuel cells convert the chemical energy stored in molecules such as hydrogen into electricity. Electrocatalysts, which typically consist of platinum nanoparticles dispersed over carbon supports, are necessary to facilitate the oxidation of hydrogen at the anode in the fuel cell. One barrier to the large-scale application of fuel cells is the decay in catalytic activity over time; therefore, a deeper understanding of deactivation mechanisms is required to allow the design of catalysts that are more robust. Now, Weilin $\mathrm{Xu}$ and colleagues in China and the US have used fluorescence microscopy to elucidate the deactivation kinetics of electrocatalysts at the single-particle level, finding that electrocatalysis-induced etching is likely to be the main cause of deactivation.

The researchers used a hydrogenconsuming platinum-catalysed probe reaction, the product of which is a fluorescent molecule. The light that was produced could be observed by fluorescence microscopy, providing a measure of catalytic activity. By alternating between monitoring the fluorescence signal and performing the electrocatalytic oxidation of hydrogen, the researchers were able to observe changes in catalytic activity on a particle-by-particle basis. They found that around $2 \%$ of the particles deactivated and then spontaneously regenerated - a feature unlikely to be seen using bulk characterization techniques. After prolonged electrocatalytic use, the smallest platinum particles could no longer be observed due to etching, which was associated with a drop in catalytic activity.

JG

Written by Nicky Dean, Elisa De Ranieri, James Gallagher and Changjun Zhang. 